\title{
REVIEW
}

\section{Is it time for home treatment of pulmonary embolism?}

\author{
Mareike Lankeit* and Stavros Konstantinides"
}

ABSTRACT: Acute pulmonary embolism (PE) is a frequent cause of death, but not all patients are at high risk of an adverse early outcome. It has been proposed that selected patients may be considered for early discharge and home treatment, but it was only recently that improved risk assessment strategies permitted advances in the identification of low-risk PE.

Clinical prediction rules, such as the Pulmonary Embolism Severity Index (PESI), and laboratory biomarkers, particularly natriuretic peptides and cardiac troponins, appeared capable of excluding severe PE and serious comorbidity.

Recently, two randomised trials and two prospective cohort studies investigated the feasibility and safety of outpatient treatment. All excluded patients with haemodynamic instability and serious comorbidity, but only one trial used a validated clinical score (PESI) for patient inclusion, and only one cohort study employed a biomarker test. Overall, 90-day outcome was favourable and the results appear promising.

To optimise patient selection, future trials will need to test simplified clinical scores combined with high-sensitivity biomarker assays, and it will have to be determined whether echocardiography and/or compression ultrasonography are also required before discharge. Furthermore, ongoing trials will show whether new oral anticoagulants are a safe and cost-effective option for managing patients out of hospital.

KEYWORDS: Biomarkers, home treatment, imaging, prognostic indicators, pulmonary embolism

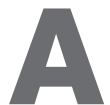
cute pulmonary embolism (PE) is a frequent cause of death and serious disability [1]. Recently, an epidemiological model derived from six European countries with a total population of 310.4 million yielded a PE incidence rate of 98 cases per 100,000 person-yrs [2]. The estimated number of fatalities related to venous thromboembolism (VTE) amounted to 370,000 , or $12 \%$ of all deaths, which corresponds to $>1$ million deaths annually in the European continent [2]. Recent registries and cohort studies suggest that $\sim 10 \%$ of all patients with acute PE die within the first 3 months after diagnosis [3, 4]. Of all patients admitted to hospitals, $1 \%$ die of acute PE and $10 \%$ of all in-hospital deaths are PE-related [2, 5, 6]. Taken together, these data demonstrate that VTE is a potentially life-threatening disease. Conversely, and importantly, case fatality rates in the acute phase vary widely, covering a range between $1 \%$ and well over $50 \%$ [7-10], thus emphasising the need for early risk stratification. Based on studies dating back to the late 1990s [11], and on the intensive research on imaging and laboratory biomarkers during the past decade [12], the guidelines of the European Society of Cardiology (ESC) on PE management, published in 2008, proposed that patients presenting without haemodynamic instability and without elevated biomarker levels or imaging findings indicating right ventricular (RV) dysfunction or myocardial injury may constitute a low-risk group; accordingly, it was suggested that these patients may be considered for early discharge and home treatment [13]. This was, however, a general position statement and by no means a clear recommendation. The reason is the lack, until very recently, of solid evidence on the appropriate selection criteria for outpatient treatment and on the exact regimen to be followed.

The present article summarises our current state of knowledge on the attempts to define low-risk PE and focuses on evolving risk assessment strategies with the use of clinical scores, imaging modalities and laboratory biomarkers. Furthermore, it reviews the accumulating evidence on outpatient treatment derived from recent prospective cohort studies and

\section{AFFILIATIONS}

*Dept of Cardiology and

Pulmonology, Heart Centre,

University of Göttingen, Göttingen,

Germany.

\#Dept of Cardiology, Democritus University of Thrace,

Alexandroupolis, Greece.

CORRESPONDENCE

S. Konstantinides

Dept of Cardiology

Democritus University of Thrace

68100 Alexandroupolis

Greece

E-mail: skonst@med.duth.gr

Received:

Dec 112011

Accepted after revision:

Feb 032012

First published online:

April 102012 
randomised trials and discusses the possible contribution of new oral anticoagulants to the simplification of PE treatment in the near future.

\section{INITIAL ANTICOAGULATION FOR PE: CURRENT REGIMENS AND STRATEGIES}

In acute PE, early deaths (within the first hours after admission) are the result of acute RV failure and cardiogenic shock [14]. After this time, the risk of death during hospitalisation is mainly determined by the potential for recurrent thromboembolic events and by the underlying disease. Thus, the treatment of PE in the acute phase must focus on two major goals: 1) the prompt reversal of RV pressure overload and failure, if present; and 2) the prevention of recurrent thromboembolism. The former goal, which is mainly achieved by thrombolysis or surgical (or transcatheter) thrombus removal, will not be discussed in this review, as "recanalisation" treatment is currently reserved for high-risk patients presenting with haemodynamic instability [15], and this situation obviously precludes early discharge and outpatient treatment of PE.

Anticoagulant treatment is administered to all patients upon clinical suspicion of acute PE, i.e. even prior to obtaining definitive confirmation of the diagnosis by imaging procedures [13]. Intravenous unfractionated heparin is the preferred mode of initial anticoagulation: 1) for patients with severe renal impairment (creatinine clearance $<20-30 \mathrm{~mL} \cdot \mathrm{min}^{-1}$ ); 2) for patients at high risk of bleeding; 3) for high-risk, hypotensive patients; and, as a rule, 4) for extremely overweight, underweight or old patients. Standardised nomograms should be used for initiation of treatment and for adjustment of heparin dosage [16]. With the exception of these circumstances, unfractionated heparin has largely been replaced by low molecularweight heparin (LMWH) or fondaparinux given subcutaneously at weight-adjusted doses. Routine anticoagulation monitoring, i.e. measurement of anti-factor Xa levels, is not necessary in patients receiving $\mathrm{LMWH}$, but it may be considered in patients with (moderate) impairment of renal function and, intermittently, during pregnancy. In these cases, anti-factor Xa levels should be determined $4 \mathrm{~h}$ after the morning injection; the proposed target range is $0.6-1.0 \mathrm{IU} \cdot \mathrm{mL}^{-1}$ for twice-daily and $1.0-2.0 \mathrm{IU} \cdot \mathrm{mL}^{-1}$ once-daily LMWH administration.

The risk of heparin-induced thrombocytopenia is highest (3-5\%) in patients who have undergone orthopaedic surgery and have received unfractionated heparin. Conversely, in medical and surgical patients receiving LMWH, the incidence is below $1 \%$ and, for patients receiving fondaparinux, the risk is negligible $[12,17]$.

Anticoagulation with unfractionated heparin or LMWH should be continued for $\geqslant 5$ days. Oral anticoagulants (vitamin $\mathrm{K}$ antagonists) should be initiated as soon as possible in all haemodynamically stable patients, preferably on the same day as heparin. Parenteral anticoagulation can be stopped as soon as the international normalised ratio has been in the therapeutic range (between 2.0 and 3.0) on two consecutive days. After a first episode of "unprovoked" PE (i.e. in the absence of transient, reversible risk factors), treatment with vitamin $\mathrm{K}$ antagonists should be continued for $\geqslant 3$ months; long-term treatment may be considered in patients with a favourable risk-to-benefit ratio $[13,18]$.

\section{WHO MIGHT BE TREATED AS AN OUTPATIENT?}

Figure 1 summarises the principal determinants of outcome in the acute phase of PE. Based on these prognostic factors (reviewed in [13]), the possible criteria for early discharge and home treatment appear theoretically easy to define, as: 1) Absence of overt right heart failure (persistent arterial hypotension or cardiogenic shock); 2) absence of RV dysfunction; 3) absence of serious comorbidity, including (but not confined to) pre-existing heart failure, chronic pulmonary disease and renal insufficiency; 4) low risk of early recurrence; and, possibly, 5) exclusion of a patent foramen ovale [19]. Further, self-evident criteria include the absence of pain requiring intravenous analgesia, the absence of hypoxaemia requiring supplemental oxygen, the absence of active bleeding, a low estimated bleeding risk upon anticoagulation and, of course, a compliant patient and a social or family background ensuring adequate therapy outside the hospital. While all the above points are more or less accepted by everyone, the challenge lies in their translation into clinical practice and, particularly, the establishment of a standardised selection process to be based on clinical parameters or scores, and (perhaps) supported by biomarkers or imaging tests.

Clinical prediction rules have been shown to be helpful in the prognostic assessment of patients with acute PE [20-23]. Of these, the Pulmonary Embolism Severity Index (PESI; table 1, left column) is the most extensively validated clinical score to date [22, 26-28]. Its major strength lies in the identification of low-risk PE (PESI classes I and II), and a recently published randomised trial successfully employed a low PESI score as an inclusion criterion for home treatment of acute PE [29]. The main limitation of the index is the fact it requires several clinical variables and is therefore not particularly simple to calculate in the setting of an emergency department. More recently [25], it was reported that reliable prognostic information can also be obtained with a simplified PESI (sPESI), which reduces the technical complexity of the original prediction rule by focusing on six equally weighed variables (table 1 , right column). In one study, the simplified PESI was at least as accurate as the imaging and biomarker criteria proposed by the ESC for identification of low risk [30], but its implications for patient management remain to be shown.

Imaging of the right ventricle (table 2) with echocardiography is capable of detecting the changes occurring in the morphology and function of the right ventricle as a result of acute pressure

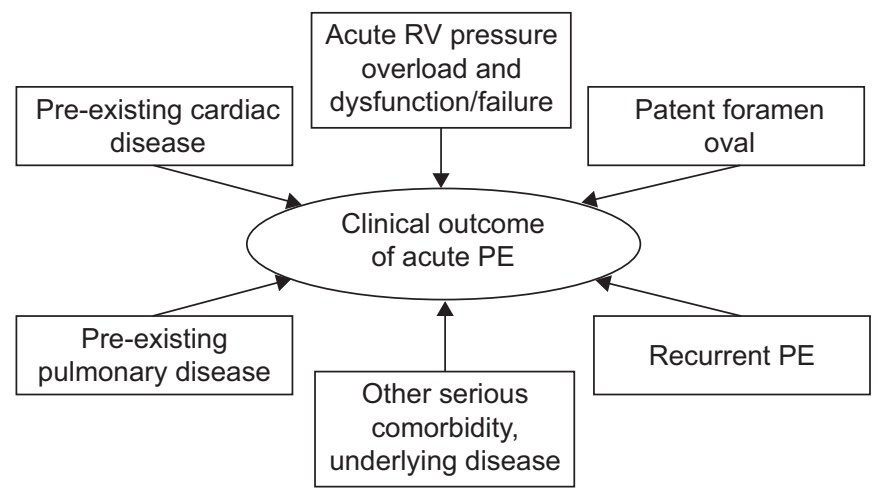

FIGURE 1. Summary of the principal determinants of outcome in the acute phase of pulmonary embolism (PE). RV: right ventricular. 
TABLE 1 Pulmonary Embolism Severity Index (PESI) and simplified PESI

\begin{tabular}{|c|c|c|c|}
\hline \multicolumn{2}{|l|}{ Original PESI [24] } & \multicolumn{2}{|l|}{ Simplified PESI [25] } \\
\hline Variable & Points & Variable & Points \\
\hline Age & 1 per year & Age $>80$ yrs & 1 \\
\hline Male sex & 10 & & \\
\hline History of cancer & 30 & History of cancer & 1 \\
\hline Pulse rate $>110$ beats $\cdot \min ^{-1}$ & 20 & Pulse rate $>110$ beats $\cdot \min ^{-1}$ & 1 \\
\hline Systolic blood pressure $<100 \mathrm{mmHg}$ & 30 & Systolic blood pressure $<100 \mathrm{mmHg}$ & 1 \\
\hline Respiratory rate $\geqslant 30$ breaths $\cdot \mathrm{min}^{-1}$ & 20 & & \\
\hline Body temperature $<36^{\circ} \mathrm{C}$ & 20 & & \\
\hline Altered mental status ${ }^{\#}$ & 60 & & \\
\hline $\mathrm{Sa}, \mathrm{O}_{2}<90 \%$ & 20 & $\mathrm{Sa}, \mathrm{O}_{2}<90 \%$ & 1 \\
\hline \multicolumn{4}{|l|}{ Class IV (106-125 points): high risk } \\
\hline Class V (>125 points): very high risk & & & \\
\hline
\end{tabular}

$\mathrm{Sa}, \mathrm{O}_{2}$ : arterial oxygen saturation. ${ }^{*}$ : disorientation, confusion or somnolence; " : patients in PESI classes I and II are collectively referred to as low-risk patients.

overload [38]. Registries and cohort studies could demonstrate an association between echocardiographic parameters of RV dysfunction and a poor in-hospital outcome [11, 39-42]. Nevertheless, the therapeutic implications of cardiac ultrasound for haemodynamically stable patients with PE remain questionable, mainly due to the poor standardisation of the echocardiographic criteria [31, 32]. Of note, a recent prospective cohort study reported that patients with acute PE, sPESI of 0 points and troponin $\mathrm{T}$ levels $<14 \mathrm{pg} \cdot \mathrm{mL}^{-1}$ by a high-sensitivity (hsTnT) assay, had an excellent short-term prognosis regardless of echocardiographic findings [43].

Four-chamber views of the heart on multidetector-row computed tomography-pulmonary angiography (CTPA; table 2), currently the preferred method for diagnosing PE, may also detect RV enlargement due to PE. A meta-analysis of two studies (with two different RV/left ventricular diameter thresholds, 1.5 and 1.0, respectively), including 191 normotensive patients with $\mathrm{PE}$, reported a $58 \%$ negative and a $57 \%$ positive value for prediction of early death [44]. The prognostic value of an enlarged RV on the computed tomography scan was recently confirmed by an international prospective cohort study [45], supporting the concept that a single test may permit both diagnosis and initial risk stratification of PE. The potential role of CTPA in helping define low-risk PE has not yet been directly investigated.

Laboratory biomarkers offer a number of theoretical advantages when used as an alternative, or in addition to, clinical prediction rules. Standardised, readily available assays yield "objective" numerical results that may assist in the quantitative assessment of RV dysfunction, myocardial injury and/or comorbidity. Natriuretic peptides are very sensitive indicators of neurohormonal activation due to ventricular overload and dysfunction. A meta-analysis of 13 studies found that $51 \%$ of
1,132 patients with acute PE had elevated brain natriuretic peptide (BNP) or N-terminal (NT)-BNP concentrations; these were associated with an increased risk of early death (OR 7.6, 95\% CI 3.4-17) and a complicated in-hospital course (OR 6.8, 95\% CI 4.4-10) [33]. A recent prospective cohort study [34] suggested that natriuretic peptides may be a useful tool for selecting candidates for home treatment thanks to their very high sensitivity and negative predictive value [46].

Elevated cardiac troponin I or T levels are found in up to $50 \%$ of patients with acute PE [47]. Studies published between 1998 and 2007 with a total of 1,985 patients were included in a metaanalysis, which showed that cardiac troponin elevation was associated with an increased risk of death (OR 5.24, 95\% CI 3.288.38 ) and major adverse events (OR 7.03, 95\% CI 2.42-20.43) in the acute phase [48]. However, another meta-analysis that excluded hypotensive patients was unable to confirm the prognostic value of circulating cardiac troponin levels [49]. Recently developed high-sensitivity assays may improve the prognostic performance of this biomarker at the low-risk end of the severity spectrum. More specifically, a derivation study showed that hsTnT was useful for excluding an adverse outcome in the acute phase of PE [50]. In a multicentre, multinational cohort of 526 normotensive patients with acute PE, hsTnT exhibited a high negative predictive value $(98 \%)$ that was comparable to that of the SPESI (99\%) [43]. Importantly, none of the patients with a sPESI of 0 points and hsTnT levels $<14 \mathrm{pg} \cdot \mathrm{mL}^{-1}$ on admission was found to have an adverse outcome within the first 30 days, supporting the notion that the combination of the two modalities can reliably identify low-risk PE.

Fatty acid-binding proteins (FABPs) are small cytoplasmic proteins that are abundant in tissues with active fatty acid metabolism, including the heart [51]. Following myocardial cell damage, heart- 
TABLE 2 Imaging and biochemical risk stratification tools in acute pulmonary embolism (PE)

Parameter assessed

Suitable for low-risk PE?

\begin{tabular}{|c|c|c|}
\hline \multicolumn{3}{|l|}{ Imaging modalities } \\
\hline \multirow[t]{4}{*}{ Echocardiography } & RV size, RV/LV ratio & Unclear/unlikely \\
\hline & $R V$ (dys)function & Moderate negative predictive value $(60 \%, 95 \% \mathrm{Cl} 55-65 \%)$ [31] \\
\hline & Systolic $P$ pa & Poorly standardised definition of RV dysfunction [32] \\
\hline & & No additive value to sPESI and hsTnT [30] \\
\hline \multirow[t]{2}{*}{ Computed tomography (CTPA) } & RV/LV ratio & Unknown \\
\hline & & Not yet tested in this context \\
\hline \multicolumn{3}{|l|}{ Laboratory biomarkers } \\
\hline \multirow[t]{2}{*}{ Cardiac troponin I, $\mathrm{T}$} & Myocardial injury, necrosis & Probably yes \\
\hline & & $\begin{array}{l}\text { Value of hsTnT (in addition or as an alternative to sPESI) confirmed in a large } \\
\text { cohort [30] }\end{array}$ \\
\hline \multirow[t]{4}{*}{ Natriuretic peptides (BNP, NT-proBNP) } & Heart (RV) failure & Probably yes \\
\hline & & Very sensitive biomarkers [33] \\
\hline & & NT-proBNP tested in a prospective cohort study of home treatment [34] \\
\hline & & But cut-off values remain unclear \\
\hline \multirow[t]{3}{*}{ H-FABP } & Myocardial injury, necrosis & Possibly yes \\
\hline & & Promising data in unselected [35] and normotensive patients [36] \\
\hline & & Assay not yet available/approved for clinical routine \\
\hline \multirow[t]{4}{*}{ GDF-15 } & Myocardial injury, heart failure & Unknown \\
\hline & & Solid pathophysiological background \\
\hline & & Promising preliminary data [37] \\
\hline & & Assay not yet available/approved for clinical routine \\
\hline
\end{tabular}

RV: right ventricular; LV: left ventricle; Ppa: pulmonary artery pressure; sPESI: simplified Pulmonary Embolism Severity Index; hsTnT: high-sensitivity troponin T; CTPA: computed tomography-pulmonary angiography; BNP: brain natriuretic peptide; NT-proBNP: N-terminal pro-brain natriuretic peptide; H-FABP: heart-type fatty acidbinding protein; GDF-15: growth differentiation factor-15.

type FABP (H-FABP) diffuses rapidly through the interstitial space; its levels in the circulation begin to rise within $30 \mathrm{~min}$ and reach a peak within $6 \mathrm{~h}$ [52]. H-FABP may provide relevant prognostic information in normotensive non-high-risk patients [53]. Cardiac expression of growth-differentiation factor (GDF)-15, a distant member of the transforming growth factor- $\beta$ cytokine family, increases sharply after pressure overload or myocardial ischaemia [54, 55]. In fact, GDF-15 might be capable of integrating information on RV dysfunction, myocardial injury and possibly comorbidity in patients with acute PE [37].

The main strengths and limitations of laboratory biomarkers, and their potential suitability for defining low-risk PE, are summarised in table 2 .

\section{OUTPATIENT TREATMENT IN PRACTICE}

Outpatient treatment is currently the standard of care for patients with deep-vein thrombosis (DVT) who have no symptomatic PE [18]. However, experts and scientific societies have, until now, carefully avoided to explicitly recommend this type of treatment in acute PE [13]. A number of observational studies, published between 2000 and 2007, reported on the early discharge and home treatment of patients with acute symptomatic PE; 11 of these studies, with a total 928 patients, were included in a metaanalysis [56]. None of the patients treated out of hospital died during the first 7 days of therapy, but the authors of the systematic review pointed out the lack of a comparator arm in several studies and, particularly, the heterogeneity of: 1) the inclusion criteria (some of which appeared rather arbitrary); 2) the duration of the hospital stay; and 3) the therapeutic regimen followed. The authors concluded that "outpatient treatment of symptomatic PE is not based on high-quality evidence" [56]. Another meta-analysis, which had been published a few months earlier and included more or less the same studies, essentially yielded similar results, although the conclusions of the authors were slightly more positive [57].

In recent years, four major prospective studies on the outpatient treatment of acute PE were published, contributing to a larger and qualitatively improved body of evidence. Their design characteristics, the patient population included, the treatment regimen(s) and their primary end-points are summarised in table 3. Two of the studies (with a total of 476 patients) were randomised, including a "standard" in-patient comparator arm $[29,59]$, while the other two (total, 449 patients) included prospective cohorts treated as outpatients $[39,58]$. All studies took care to exclude patients with haemodynamic instability, those with serious comorbidity requiring hospitalisation or expected to aggravate prognosis, and those lacking the necessary compliance and the family and social background to support home treatment. However, only one randomised trial used a standardised, validated clinical score (the PESI in its original form) to select low-risk patients [29], and only one cohort study employed a biomarker test (NT-proBNP) to assist in the exclusion of acute heart failure [34]. Overall, the proportion of screened patients who were ultimately included and analysed 


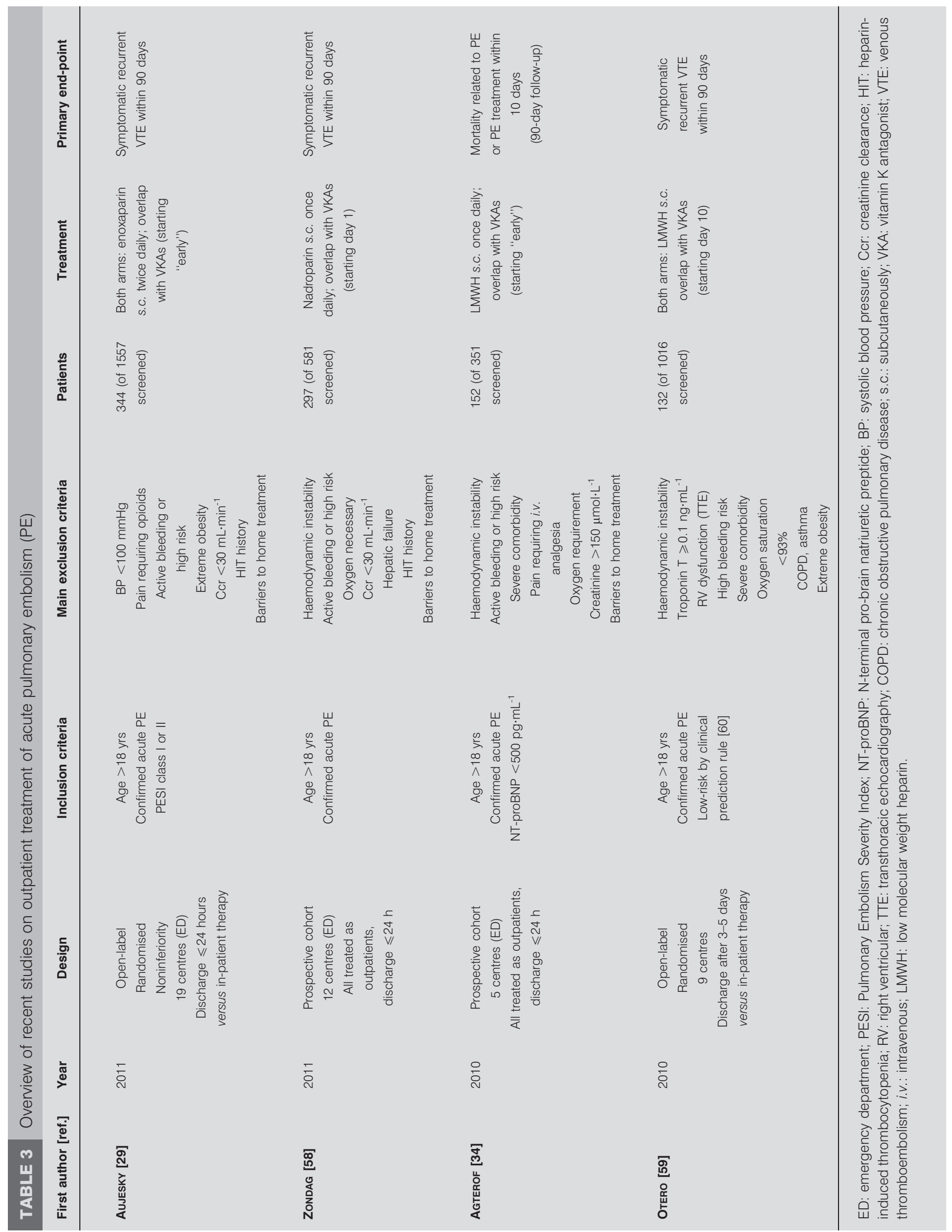


was (only) $26 \%$ (table 3), with a range between $13 \%$ [59] and $51 \%$ [39]; this fact emphasises the persisting uncertainty regarding the appropriate inclusion and exclusion criteria. In general, the patients' outcome at 90 days appeared favourable and the results can be regarded as promising. For example, the latest randomised trial by AUJESKY et al. [29] reported a $0.6 \%$ rate of recurrent VTE and a $0.6 \%$ rate of death in the outpatient arm. In the prospective cohort study by ZONDAG et al. [58], the 3month death rate was $1 \%(95 \%$ CI $0.2-2.9 \%$; all deaths reportedly unrelated to PE) and the recurrence rate of VTE was 2\% (95\% CI $0.8-4.3 \%)$. In the second cohort study, conducted by AGTEROF et al. [34], no early deaths or VTE recurrence were reported. Conversely, there was also an alarming observation, namely the premature discontinuation of the randomised trial by OTERO et al. [59] because of a $2.8 \%$ mortality in the early discharge group as opposed to the lack of early deaths in the in-patient group.

Have these four recent studies significantly advanced our state of knowledge regarding the feasibility and safety of home treatment in acute PE? Definitely. Have they provided conclusive evidence to standardise the selection of low-risk patients as candidates for early discharge? Hardly. In fact, a number of important issues remain to be resolved by future studies, as follows.

1) Can the use of a simple, "user-friendly" clinical score such as the sPESI [25] reproduce the promising results of the randomised trial that employed the original, rather complex version of the PESI [29]?

2) Does the combination of a simple clinical prediction rule with a high-sensitivity biomarker assay offer additional safety in the selection of patients [43]?

3) Is an echocardiogram on admission necessary in order to exclude large thrombi in the right heart cavities (presumably the cause of one fatal cardiac arrest in the randomised trial by OTERO et al. [59]), or the presence of a patent foramen ovale [19]?

4) Is compression ultrasonography of the leg veins necessary to exclude concomitant DVT, which was recently confirmed as an independent predictor of early recurrence and death [61]?

\section{THE FUTURE OF OUTPATIENT TREATMENT}

Dabigatran, a direct oral thrombin inhibitor, has been compared with warfarin in patients with acute symptomatic VTE. The RE-COVER study, published in 2009, was a doubleblind, double-dummy, noninferiority, randomised trial, comparing 6 months of treatment with dabigatran, at a fixed dose of $150 \mathrm{mg}$ twice daily, with dose-adjusted warfarin therapy, after initial parenteral anticoagulation in patients with symptomatic VTE [62]. Recurrent VTE occurred in 30 patients (2.4\%) given dabigatran during the treatment period, as compared with 27 $(2.1 \%)$ patients given warfarin $(\mathrm{p}<0.001$ for noninferiority). Major bleeding was reported in $20(1.6 \%)$ patients allocated to the dabigatran group compared with $24(1.9 \%)$ patients allocated to the control group. The RE-COVER study thus showed that dabigatran, given after a "not-so-brief" period (mean 11 days) of parenteral anticoagulation, was as effective and as safe as warfarin for the treatment of VTE.

Rivaroxaban, an oral factor Xa inhibitor, has been compared with standard treatment in patients with acute VTE. The arm of the study including patients with acute DVT, EINSTEIN-DVT, was published in 2010; this was an open-label, randomised, event-driven, noninferiority study that compared oral rivaroxaban alone with subcutaneous enoxaparin followed by a vitamin $\mathrm{K}$ antagonist for 3, 6 or 12 months [63]. Recurrent VTE occurred in $36(2.1 \%)$ patients given rivaroxaban as compared with $51(3.0 \%)$ given standard therapy $(p<0.001$ for noninferiority). Major or clinically significant bleeding occurred in 139 $(8.1 \%)$ patients in the rivaroxaban group and in $138(8.1 \%)$ of those in the control group. EINSTEIN-DVT thus showed that rivaroxaban, given as a single oral agent from the beginning, is as effective and safe as subcutaneous LMWH followed by a vitamin $\mathrm{K}$ antagonist. The results of the study arm focusing on acute PE (EINSTEIN-PE) were published recently [64]. As in the DVT arm, rivaroxaban was noninferior to standard therapy. Major or clinically relevant nonmajor bleeding also occurred with similar frequency in rivaroxaban patients compared with those under standard therapy; however, major bleeding was less frequent under rivaroxaban $(1.1 \%$ versus $2.2 \%$; $=0.003)$. In particular, intracranial bleeding occurred in one rivaroxaban patient compared with 10 patients receiving standard therapy. The single oral drug approach is also being evaluated in an ongoing trial testing the factor Xa inhibitor apixaban (AMPLIFY; NCT00643201). The potential of new oral anticoagulants for outpatient treatment of low-risk PE can directly be addressed in prospective management (cohort) studies.

\section{CONCLUSIONS AND OUTLOOK}

For many years, the lack of high-quality data regarding the definition of low-risk PE and the selection of appropriate candidates for home treatment has precluded clear-cut, evidencebased recommendations by experts and scientific societies. Fortunately, significant progress is now being made in the field. Risk stratification algorithms have been refined and simplified, and the first large randomised trials and prospective cohort (management) studies supported the feasibility and safety of home treatment in selected cases. From the most recent data discussed in the present review, it appears that the combination of a validated clinical score with a high-sensitivity troponin assay might offer the highest degree of safety in identifying possible candidates for early discharge and home treatment. In this regard, the results of ongoing or completed trials testing new oral anticoagulants are also being expected, as they will show whether these agents can provide a safe, user-friendly and cost-effective alternative to standard regimens for managing patients out of hospital. These advances may radically change the management of acute PE at the low-risk end of the severity spectrum in the near future.

\section{STATEMENT OF INTEREST}

A statement of interest for S. Konstantinides can be found at www.erj. ersjournals.com/site/misc/statements.xhtml

\section{REFERENCES}

1 US Dept of Health and Human Services. Acting Surgeon General Issues 'Call to Action to Prevent Deep Vein Thrombosis and Pulmonary Embolism, 2008. www.surgeongeneral.gov/news/ pressreleases/pr20080915.html Date last updated: September 15, 2008. Date last accessed: May 23, 2012.

2 Cohen AT, Agnelli G, Anderson FA, et al. Venous thromboembolism (VTE) in Europe. The number of VTE events and associated morbidity and mortality. Thromb Haemost 2007; 98: 756-764. 
3 Aujesky D, Jimenez D, Mor MK, et al. Weekend versus weekday admission and mortality after acute pulmonary embolism. Circulation 2009; 119: 962-968.

4 Laporte S, Mismetti P, Decousus H, et al. Clinical predictors for fatal pulmonary embolism in 15,520 patients with venous thromboembolism: findings from the Registro Informatizado de la Enfermedad TromboEmbolica venosa (RIETE) Registry. Circulation 2008; 117: 1711-1716.

5 Cohen AT, Edmondson RA, Phillips MJ, et al. The changing pattern of venous thromboembolic disease. Haemostasis 1996; 26: 65-71.

6 Lindblad B, Sternby NH, Bergqvist D. Incidence of venous thromboembolism verified by necropsy over 30 years. BMJ 1991; 302: 709-711.

7 Goldhaber SZ, Visani L, De Rosa M. Acute pulmonary embolism: clinical outcomes in the International Cooperative Pulmonary Embolism Registry (ICOPER). Lancet 1999; 353: 1386-1389.

8 Kasper W, Konstantinides S, Geibel A, et al. Management strategies and determinants of outcome in acute major pulmonary embolism: results of a multicenter registry. J Am Coll Cardiol 1997; 30: 1165-1171.

9 British Thoracic Society. Optimum duration of anticoagulation for deep-vein thrombosis and pulmonary embolism. Research Committee of the British Thoracic Society. Lancet 1992; 340: 873-876.

10 Carson JL, Kelley MA, Duff A, et al. The clinical course of pulmonary embolism. N Engl J Med 1992; 326: 1240-1245.

11 Kasper W, Konstantinides S, Geibel A, et al. Prognostic significance of right ventricular afterload stress detected by echocardiography in patients with clinically suspected pulmonary embolism. Heart 1997; 77: 346-349.

12 Konstantinides S. Clinical practice. Acute pulmonary embolism. N Engl J Med 2008; 359: 2804-2813.

13 Torbicki A, Perrier A, Konstantinides SV, et al. Guidelines on the diagnosis and management of acute pulmonary embolism: The Task Force for the Diagnosis and Management of Acute Pulmonary Embolism of the European Society of Cardiology (ESC). Eur Heart J 2008; 29: 2276-2315.

14 Stein PD, Henry JW. Prevalence of acute pulmonary embolism among patients in a general hospital and at autopsy. Chest 1995; 108: 978-981.

15 Jaff MR, McMurtry MS, Archer SL, et al. Management of massive and submassive pulmonary embolism, iliofemoral deep vein thrombosis, and chronic thromboembolic pulmonary hypertension: a scientific statement from the American Heart Association. Circulation 2011; 123: $1788-1830$.

16 Raschke RA, Reilly BM, Guidry JR, et al. The weight-based heparin dosing nomogram compared with a "standard care" nomogram. A randomized controlled trial. Ann Intern Med 1993; 119: 874-881.

17 Arepally GM, Ortel TL. Clinical practice. Heparin-induced thrombocytopenia. N Engl J Med 2006; 355: 809-817.

18 Kearon C, Kahn SR, Agnelli G, et al. Antithrombotic therapy for venous thromboembolic disease: American College of Chest Physicians Evidence-Based Clinical Practice Guidelines (8th Edn). Chest 2008; 133: 454S-545S.

19 Konstantinides S, Geibel A, Kasper W, et al. Patent foramen ovale is an important predictor of adverse outcome in patients with major pulmonary embolism. Circulation 1998; 97: 1946-1951.

20 Jimenez D, Yusen RD. Prognostic models for selecting patients with acute pulmonary embolism for initial outpatient therapy. Curr Opin Pulm Med 2008; 14: 414-421.

21 Davies CW, Wimperis J, Green ES, et al. Early discharge of patients with pulmonary embolism: a two-phase observational study. Eur Respir J 2007; 30: 708-714.

22 Aujesky D, Obrosky DS, Stone RA, et al. Derivation and validation of a prognostic model for pulmonary embolism. Am J Respir Crit Care Med 2005; 172: 1041-1046.
23 Wicki J, Perrier A, Perneger TV, et al. Predicting adverse outcome in patients with acute pulmonary embolism: a risk score. Thromb Haemost 2000; 84: 548-552.

24 Aujesky D, Roy PM, Le Manach CP, et al. Validation of a model to predict adverse outcomes in patients with pulmonary embolism. Eur Heart J 2006; 27: 476-481.

25 Jimenez D, Aujesky D, Moores L, et al. Simplification of the pulmonary embolism severity index for prognostication in patients with acute symptomatic pulmonary embolism. Arch Intern Med 2010; 170: 1383-1389.

26 Donze J, Le Gal G, Fine MJ, et al. Prospective validation of the Pulmonary Embolism Severity Index. A clinical prognostic model for pulmonary embolism. Thromb Haemost 2008; 100: 943-948.

27 Aujesky D, Roy PM, Le Manach CP, et al. Validation of a model to predict adverse outcomes in patients with pulmonary embolism. Eur Heart J 2006; 27: 476-481.

28 Aujesky D, Obrosky DS, Stone RA, et al. A prediction rule to identify low-risk patients with pulmonary embolism. Arch Intern Med 2006; 166: 169-175.

29 Aujesky D, Roy PM, Verschuren F, et al. Outpatient versus inpatient treatment for patients with acute pulmonary embolism: an international, open-label, randomised, non-inferiority trial. Lancet 2011; 378: 41-48.

30 Lankeit M, Gomez V, Wagner C, et al. A strategy combining imaging and laboratory biomarkers in comparison to a simplified clinical score for risk stratification of patients with acute pulmonary embolism. Chest 2012; 141: 916-922.

31 Sanchez O, Trinquart L, Colombet I, et al. Prognostic value of right ventricular dysfunction in patients with haemodynamically stable pulmonary embolism: a systematic review. Eur Heart J 2008; 29: 1569-1577.

32 ten Wolde M, Sohne M, Quak E, et al. Prognostic value of echocardiographically assessed right ventricular dysfunction in patients with pulmonary embolism. Arch Intern Med 2004; 164: 1685-1689.

33 Klok FA, Mos IC, Huisman MV. Brain-type natriuretic peptide levels in the prediction of adverse outcome in patients with pulmonary embolism: a systematic review and meta-analysis. Am J Respir Crit Care Med 2008; 178: 425-430.

34 Agterof MJ, Schutgens RE, Snijder RJ, et al. Out of hospital treatment of acute pulmonary embolism in patients with a low NT-proBNP level. J Thromb Haemost 2010; 8: 1235-1241.

35 Puls M, Dellas C, Lankeit M, et al. Heart-type fatty acid-binding protein permits early risk stratification of pulmonary embolism. Eur Heart J 2007; 28: 224-229.

36 Dellas C, Puls M, Lankeit M, et al. Elevated heart-type fatty acidbinding protein levels on admission predict an adverse outcome in normotensive patients with acute pulmonary embolism. J Am Coll Cardiol 2010; 55: 2150-2157.

37 Lankeit M, Kempf T, Dellas C, et al. Growth differentiation factor15 for prognostic assessment of patients with acute pulmonary embolism. Am J Respir Crit Care Med 2008; 177: 1018-1025.

38 Konstantinides S. Pulmonary embolism: impact of right ventricular dysfunction. Curr Opin Cardiol 2005; 20: 496-501.

39 Kucher N, Goldhaber SZ. Management of massive pulmonary embolism. Circulation 2005; 112: e28-e32.

40 Grifoni S, Olivotto I, Cecchini P, et al. Short-term clinical outcome of patients with acute pulmonary embolism, normal blood pressure, and echocardiographic right ventricular dysfunction. Circulation 2000; 101: 2817-2822.

41 Ribeiro A, Lindmarker P, Juhlin-Dannfelt A, et al. Echocardiography Doppler in pulmonary embolism: right ventricular dysfunction as a predictor of mortality rate. Am Heart J 1997; 134: 479-487.

42 Goldhaber SZ, Haire WD, Feldstein ML, et al. Alteplase versus heparin in acute pulmonary embolism: randomised trial assessing right-ventricular function and pulmonary perfusion. Lancet 1993; 341: 507-511. 
43 Lankeit M, Jimenez D, Kostrubiec M, et al. Predictive value of the highly sensitive troponin $\mathrm{T}$ assay and the simplified Pulmonary Embolism Severity Index in hemodynamically stable patients with acute pulmonary embolism: a prospective validation study. Circulation 2011; 124: 2716-2724.

44 Sanchez O, Trinquart L, Colombet I, et al. Prognostic value of right ventricular dysfunction in patients with haemodynamically stable pulmonary embolism: a systematic review. Eur Heart J 2008; 29: 1569-1577.

45 Becattini C, Agnelli G, Vedovati MC, et al. Multidetector computed tomography for acute pulmonary embolism: diagnosis and risk stratification in a single test. Eur Heart J 2011; 32: 1657-1663.

46 Kucher N, Goldhaber SZ. Cardiac biomarkers for risk stratification of patients with acute pulmonary embolism. Circulation 2003; 108: 2191-2194.

47 Korff S, Katus HA, Giannitsis E. Differential diagnosis of elevated troponins. Heart 2006; 92: 987-993.

48 Becattini C, Vedovati MC, Agnelli G. Prognostic value of troponins in acute pulmonary embolism: a meta-analysis. Circulation 2007 116: 427-433.

49 Jimenez D, Uresandi F, Otero $\mathrm{R}$, et al. Troponin-based risk stratification of patients with acute nonmassive pulmonary embolism: systematic review and metaanalysis. Chest 2009; 136: 974-982.

50 Lankeit M, Friesen D, Aschoff J, et al. Highly sensitive troponin T assay in normotensive patients with acute pulmonary embolism. Eur Heart J 2010; 31: 1836-1844.

51 Storch J, Thumser AE. The fatty acid transport function of fatty acid-binding proteins. Biochim Biophys Acta 2000; 1486: 28-44.

52 Alhadi HA, Fox KA. Do we need additional markers of myocyte necrosis: the potential value of heart fatty-acid-binding protein. QJM 2004; 97: 187-198.

53 Dellas C, Puls M, Lankeit M, et al. Elevated heart-type fatty acidbinding protein levels on admission predict an adverse outcome in normotensive patients with acute pulmonary embolism. J Am Coll Cardiol 2010; 55: 2150-2157.
54 Kempf T, Eden M, Strelau J, et al. The transforming growth factor-beta superfamily member growth-differentiation factor-15 protects the heart from ischemia/reperfusion injury. Circ Res 2006; 98: 351-360.

$55 \mathrm{Xu}$ J, Kimball TR, Lorenz JN, et al. GDF15/MIC-1 functions as a protective and antihypertrophic factor released from the myocardium in association with SMAD protein activation. Circ Res 2006; 98: 342-350.

56 Squizzato A, Galli M, Dentali F, et al. Outpatient treatment and early discharge of symptomatic pulmonary embolism: a systematic review. Eur Respir J 2009; 33: 1148-1155.

57 Janjua M, Badshah A, Matta F, et al. Treatment of acute pulmonary embolism as outpatients or following early discharge. A systematic review. Thromb Haemost 2008; 100: 756-761.

58 Zondag W, Mos IC, Creemers-Schild D, et al. Outpatient treatment in patients with acute pulmonary embolism: the Hestia Study. J Thromb Haemost 2011; 9: 1500-1507.

59 Otero R, Uresandi F, Jimenez $\mathrm{D}$, et al. Home treatment in pulmonary embolism. Thromb Res 2010; 126: e1-e5.

60 Uresandi F, Otero R, Cayuela A, et al. Escala de riesgo de eventos adversos a corto plazo en pacientes con bromboembolia pulmonar [A clinical prediction rule for identifying short-term risk of adverse events in patients with pulmonary thromboembolism]. Arch Bronconeumol 2007; 43: 617-622.

61 Jimenez D, Aujesky D, Diaz G, et al. Prognostic significance of deep vein thrombosis in patients presenting with acute symptomatic pulmonary embolism. Am J Respir Crit Care Med 2010; 181: 983-991.

62 Schulman S, Kearon C, Kakkar AK, et al. Dabigatran versus warfarin in the treatment of acute venous thromboembolism. N Engl J Med 2009; 361: 2342-2352.

63 Bauersachs R, Berkowitz SD, Brenner B, et al. Oral rivaroxaban for symptomatic venous thromboembolism. N Engl J Med 2010; 363: 2499-2510.

64 Buller HR, Prins MH, Lensin AW, et al. Oral rivaroxaban for the treatment of symptomatic pulmonary embolism. N Engl J Med 2012; 366: 1287-1297. 